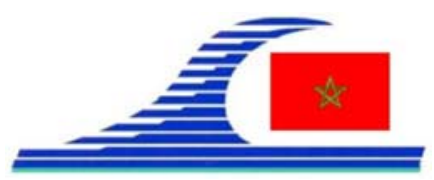

Conférence Méditerranéenne Côtière et Maritime EDITION 2, TANGER, MAROC (2011)

Coastal and Maritime Mediterranean Conference

Disponible en ligne - http://www.paralia.fr - Available online

\title{
Evolution morphodynamique et morphosédimentaire du littoral méditerranéen de Oued Laou (Tétouan, Maroc)
}

\author{
Brahim EL MOUTCHOU ${ }^{1}$, Latifa EL FADEL ${ }^{1}$, Khalil EL HAJJAJI ${ }^{1}$
}

1. Dépt. de Géologie, Faculté des Sciences. BP. 2121, M’hanech. 2. 93000 Tétouan, Maroc. brahimelmoutchou@yahoo.fr

\section{Résumé :}

L'étude morphologique et l'analyse des données historiques des sites situés sur le littoral méditerranéen de la région de Oued Laou, nous a permis d'apprécier les conditions naturelles, les tendances évolutives et les facteurs régissant l'ensembles de phénomènes enregistrés à ce niveau.

D’une façon générale, les sites étudiés présentent une tendance évolutive régressive, dont les facteurs la contrôlant sont soit d’ordre local et/ou régional.

Mots-Clés :

Evolution historique - Dérive littorale - Oued Laou - Méditerranée - Maroc

\section{Introduction}

Les écosystèmes côtiers sont des interfaces en perpétuel changement dans le temps et dans l'espace. Ils sont soumis à des influences continentales et marines. De ce fait, ils sont des exutoires naturels des bassins versants continentaux et reçoivent par lessivage des sols, des eaux enrichies des divers produits de l'érosion. Inversement à ce constat des faits, le littoral de Oued Laou évolue en contexte régressif dont il faut, connaitre les causes responsables.

\section{Caractères généraux de la région étudiée}

Le littoral méditerranéen de Oued Laou orienté NW-SE, s’étend sur une longueur d'environ $10 \mathrm{~km}$ et une largeur variable allant de 0 à $150 \mathrm{~m}$. La pente est en général, inférieure à $4 \%$.

Ce domaine côtier (Région de Oued Laou) est limité à l'Est par la mer Méditerranée et à l'Ouest, Sud et au Nord par les formations paléozoïques du Rif interne. Il se caractérise par un climat de type méditerranéen à été chaud et sec et hiver aux précipitations souvent brutales (EL GHARBAOUI, 1981), (figure. 1).

Les caractéristiques climatiques de la région sont : Une température moyenne annuelle d'environ $19^{\circ} \mathrm{C}$, une pluviométrie moyenne annuelle d'environ $600 \mathrm{~mm} / \mathrm{an}$, répartie sur 90 jours et des vents dominés pour l'essentiel par ceux du secteur Est.

Les principaux cours d'eau de la région (Laou et Assresse) sont à régime torrentiel (EL FADEL, 2006 ; EL MOUTCHOU \& EL FADEL, 2005). 
La connaissance de la Mer :

un vecteur du développement durable en Méditerranée

Le contexte géomorphologique présente des zones hautes (Caps), des zones basses et un arrière-pays dominé par les formations du domaine interne.

Le littoral méditerranéen de la région de Oued Laou borde la partie méridionale du domaine interne rifain. Ce dernier, constitue avec les cordillères bétiques occidentales, l'arc de Gibraltar (DURAND DELGA et al., 1960-1962 ; CHALOUAN 1986).

Le contexte hydrodynamique sur le littoral méditerranéen (LACOMBE \& RICHEZ, 1982), est dominé par une courantologie dominée par les courants résultants des fortes houles d'Est avec une amplitude d'environ 5,5m et une période de 5 à 9,5sec et une faible onde de marée, de type semi-diurne, avec un marnage moyen en vive eau d'environ 0,8 à $1 \mathrm{~m}$.

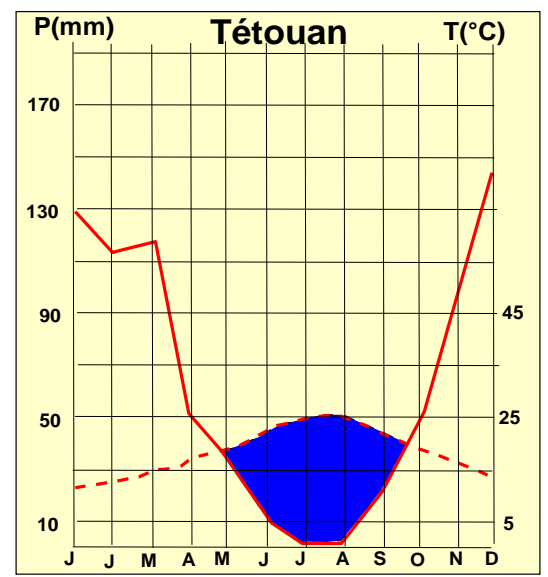

Figure 1. Caractéristiques climatiques du littoral de la région de Oued Laou.

\section{Caractérisation sédimentologique le long du littoral de Oued Laou}

La caractérisation de l'évolution morphosédimentaire du littoral de Oued Laou a été effectuée par l'analyse des échantillons superficiels de la plage en fonction du changement de faciès granulométrique (EL FADEL, 2006 ; EL MOUTCHOU, 1995 et 2003 ; EL MOUTCHOU \& EL FADEL, 2005 ; NACHITE et al., 2004).

Le traitement des échantillons sableux (Mission d'avril 2006), a montré la dominance d'un faciès sableux moyens bioclastiques. L'analyse granulométrique détaillée a permis la mise en évidence :

- d'une fraction terrigène formée de quartz, micas et de lithoclastes.

- d'une fraction bioclastique brisé ou intact, essentiellement formée de Lamillibranches et des Gastéropodes.

De cet état de lieu, se dégage (figure. 2) :

- un gradient granulométrique décroissant du SE vers le NW, traduisant une dérive littorale du SE vers le NW.

- un gradient granulométrique de l'Est vers l'Ouest, traduisant le brassage par les courants transversaux (Courants induits par les vagues et le jeu de marée en plus de l'impact fluvial). 


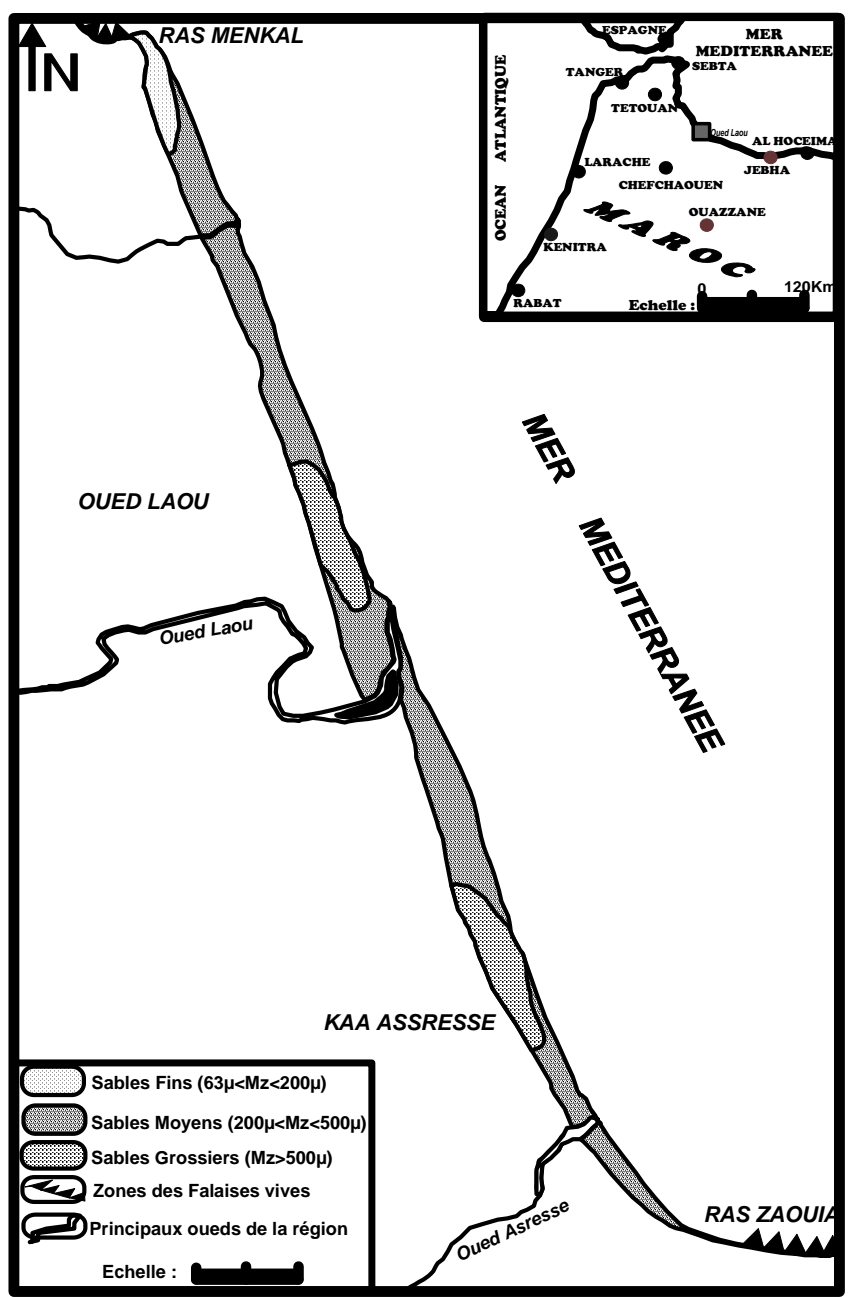

Figure 2. Carte de la répartition superficielle des matériaux sur le littoral de Oued Laou.

\section{Evolution historique de la ligne de rivage le long du littoral de Oued Laou}

Le comportement du trait de côte a été étudié à travers la comparaison des documents cartographiques : Cartes topographiques de 1937 et 1971 et photographies aériennes des missions de 1958 et 1994 (EL FADEL, 2006; EL MOUTCHOU, 1995; EL MOUTCHOU \& EL FADEL, 2005 ; EL FADEL et al., 2007).

L'analyse détaillée de ces documents, nous a permis d'apprécier et d'évaluer les différents types de modifications qu'ont subi les différentes unités morphologiques de ce secteur pendant trois tranches de temps continues et couvrant respectivement avec : Une période de 20 ans s'étalant de 1937 à 1957, une période de 13 ans s'étalant de 1958 à 1970 et une période de 24 ans s’étalant de 1971 à 1994.

Le bilan évolutif du comportement du trait de côte sur le littoral méditerranéen de Oued Laou révèle (tableau. 1 et figures 3 et 4). 
La connaissance de la Mer :

un vecteur du développement durable en Méditerranée

Tableau 1. Bilan évolutif du comportement du trait de côte le long du littoral de Oued Laou.

\begin{tabular}{lll}
\hline & Taux de recul & Taux moyen de recul \\
\hline de 1937 à 1957 & $.40 \mathrm{~m} / 20$ ans & $-2,00 \mathrm{~m} / \mathrm{an}$ \\
de 1958 à 1970 & $.40 \mathrm{~m} / 13$ ans & $-3,08 \mathrm{~m} / \mathrm{an}$ \\
de 1971 à 1994 & $.90 \mathrm{~m} / 24$ ans & $-3,75 \mathrm{~m} / \mathrm{an}$ \\
de 1937 à 1994 & $.170 \mathrm{~m} / 57$ ans & $-2,98 \mathrm{~m} / \mathrm{an}$ \\
\hline
\end{tabular}

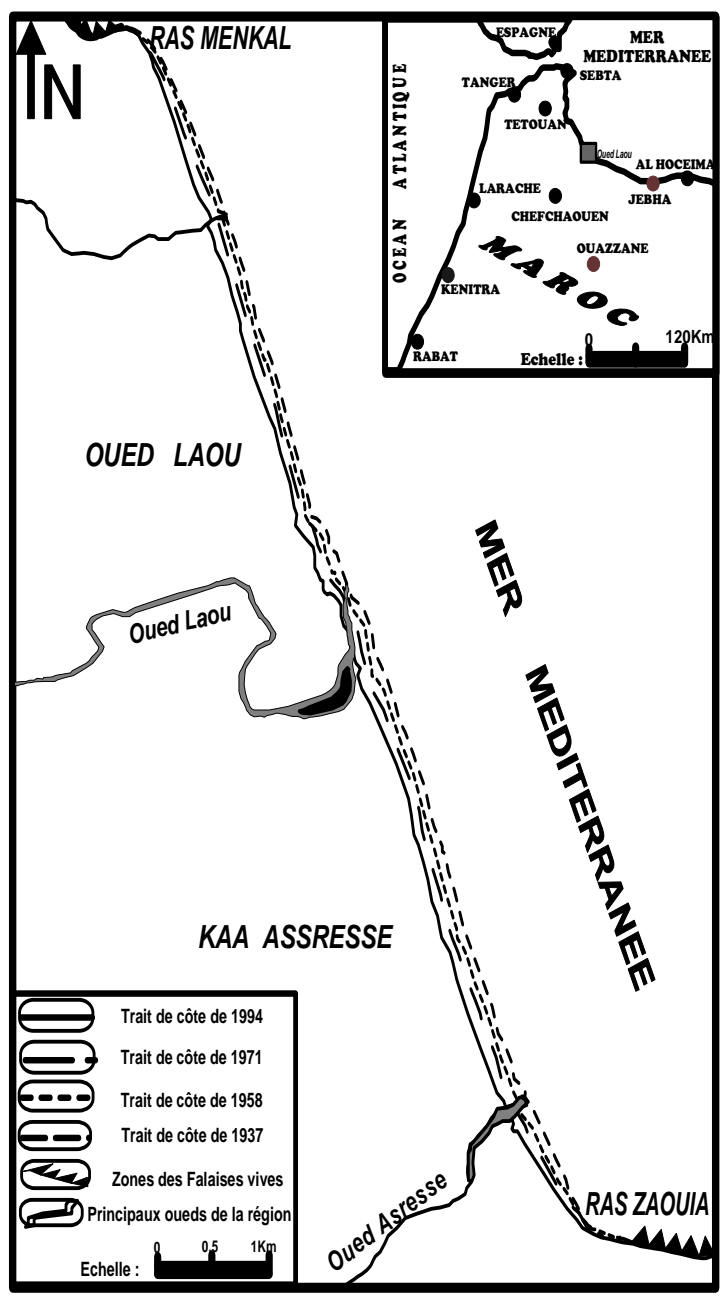

Figure 3. Carte du comportement de la ligne de rivage sur le littoral de Oued Laou.

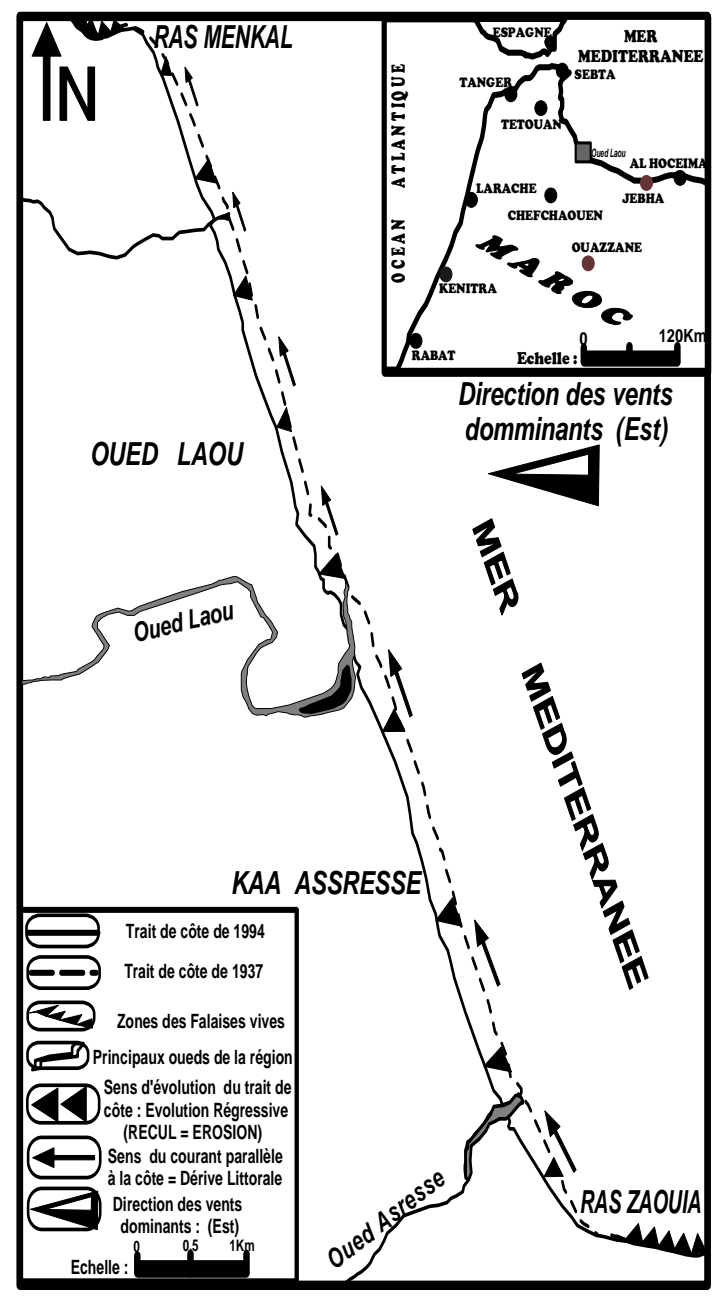

Figure 4. Schéma de la dynamique littorale le long du littoral de Oued Laou.

Les profils ainsi, réalisés sur la base de l'analyse des cartes topographiques et des photographies aériennes, montre un recul généralisé du trait de côte d'environ -170 mètres linéaires sur 57 ans avec une moyenne annuelle d'environ -3 mètres linéaires/an. Cette tendance évolutive générale vers l'érosion (figure. 4) est due à la conjugaison des effets de plusieurs facteurs dont les plus importants sont : 
A best knowledge of the Sea:

- l'élévation du niveau marin,

- le contexte océanologique dominé par les fortes houles, les tempêtes et les courants induits par effet de houle en plus des interactions fluvio-marines,

- les effets de néotectoniques au niveau de la grande plaine de Oued Laou par la réactivation du réseau de faille parallèle et perpendiculaire à la côte,

- les actions anthropiques avec l'urbanisation accrue du littoral au dépend des unités morphologiques de la plage et l'extraction abusive des sables et des galets.

\section{Conclusion générale}

En conclusion, le contexte morpho-dynamique et historique de la frange littorale méditerranéenne de Oued Laou, montre :

- un brassage mécanique, des sédiments littoraux, suite à la superposition dans le temps des effets de deux dynamiques différentes : Marine et fluviale.

- une évolution à composante (E-W), avec une répartition des sables selon un gradient décroissant Est - Ouest, régi par les courants perpendiculaires à la côte.

- une évolution à composante (SE-NW), avec un gradient décroissant des sables du SE vers le NW, régi par la dérive littorale (SE-NW).

- une tendance générale à l’érosion avec un recul de l’ordre -3 mètres linéaires/an.

\section{Références Bibliographiques}

CHALOUAN A. (1986). Les nappes ghomarides (Rif septentrional Maroc). Un terrain varisque dans la chaîne alpine. Thèse Doc. Sc., Univ. Strasbourg, 317 p.

DURAND DELGA M., HOTTINGER L., MARCAIS J., MATTAUER M., MILLIARD Y., SUTER G. (1960-1962). Données actuelles sur la structure du Rif. BSGF, t. 1, pp 399-422.

EL FADEL L. (2006). Evolution morpho-dynamique et analyse morphostructurale du littoral méditerranéen marocain de Oued Laou (Provinces de Tétouan et Chefchaouen Maroc Nord Occidental). Mémoire de DESA. Univ. Abdelmalek Essaâdi, 76 p.

EL FADEL L., EL MOUTCHOU B., OUAZANI TOUHAMI A., OUAZZANI TOUHAMI M. (2007). Etude morphodynamique et morphostructurale du litoral méditerranéen de la région de Oued Laou (Provinces de Tétouan et Chefchaouen Maroc Nord Occidental). Actes des $4^{\text {èmes }}$ Jour. Intern. des Géosciences de l'Environnement. juin 2007, 176 p.

EL MOUTCHOU B. (1995). Dynamique côtière actuelle et évolution morphodynamique de la frange littorale entre M'diq et Oued Laou (Province de Tétouan, Maroc Nord Occidental). Thèse de Doctorat de $3^{\circ}$ cycle, Univ Mohamed V, Fac. Sc. Rabat, 165 p.

EL MOUTCHOU B. (2003). Dynamique côtière et évolution spatio-temporelle de la frange littorale méditerranéenne entre Fnideq et Martil (Province Tétouan, Maroc). Bull. CIESM, Série n 18, pp 35-37. 
La connaissance de la Mer :

un vecteur du développement durable en Méditerranée

EL MOUTCHOU B., EL FADEL L. (2005). Le littoral Méditerranéen de Tétouan entre Sebta et Martil : Evolution et Impacts des aménagements littoraux (Province Tétouan, Maroc Nord Occidental). $3^{\text {ème }}$ J. Géosiences Environts. Publ. Fac. Sc. El Jadida.

LACOMBE H., RICHEZ C. (1982). The regime of the strait of Gibraltar. In: Hydrodynamics of semi-enclosed seas, edited by J.C.J. Nihoul, Elsevier, Amesterdam, pp 13-73.

NACHITE D., EL MOUTCHOU B., ANFUSO G., BENAVENTE J., BELLO E., MACIAS A. (2004). Morfología y evolución reciente del litoral entre Fnideq y M'diq (Tetuán, NE de Marruecos). GEOGACETA, 35, pp 43-46. 